\title{
A SYSTEM OF IMPULSIVE DEGENERATE NONLINEAR PARABOLIC FUNCTIONAL-DIFFERENTIAL INEQUALITIES
}

\author{
LUDWIK BYSZEWSKI \\ Cracow University of Technology \\ Institute of Mathematics \\ Cracow 31-155, POLAND
}

(Received April, 1994; revised June, 1994)

\begin{abstract}
A theorem about a system of strong impulsive degenerate nonlinear parabolic functional-differential inequalities in an arbitrary parabolic set is proved. As a consequence of the theorem, some theorems about impulsive degenerate nonlinear parabolic differential inequalities and the uniqueness of a classical solution of an impulsive degenerate nonlinear parabolic differential problem are established.
\end{abstract}

Key words: Impulsive Parabolic Problems, Diagonal Systems, Functional-Differential Inequalities, Impulsive Conditions, Uniqueness Criterion, Arbitrary Parabolic Sets.

AMS (MOS) subject classifications: $35 \mathrm{~K} 65,35 \mathrm{R} 10,35 \mathrm{~K} 85,35 \mathrm{~K} 60,35 \mathrm{~K} 99$.

\section{Introduction}

In this paper we prove a theorem about strong inequalities for the following diagonal system of degenerate nonlinear parabolic functional-differential inequalities

$$
\begin{gathered}
F_{i}\left(t, x, u(t, x), u_{t}^{i}(t, x), u_{x}^{i}(t, x), u_{x x}^{i}(t, x), u\right) \\
>F_{i}\left(t, x, v(t, x), v_{t}^{i}(t, x), v_{x}^{i}(t, x), v_{x x}^{i}(t, x), v\right) \quad(i=1, \ldots, m),
\end{gathered}
$$

where $(t, x) \in D \backslash \bigcup_{j=1}^{s}\left(\left\{t_{j}\right\} \times \mathbb{R}^{n}\right), t_{0}<t_{1}<\ldots<t_{s}<t_{0}+T$ and $D$ is a relatively arbitrary set more general than the cylindrical domain $\left(t_{0}, t_{0}+T\right) \times \Omega_{0} \subset \mathbb{R}^{n+1}$. In the expressions

$$
F_{i}\left(t, x, w(t, x), w_{t}^{i}(t, x), w_{x}^{i}(t, x), w_{x x}^{i}(t, x), w\right) \quad(i=1, \ldots, m)
$$

the symbol $w$ denotes a function

$$
w: \widetilde{D} \ni(t, x) \rightarrow w(t, x)=\left(w^{1}(t, x), \ldots, w^{m}(t, x)\right) \in \mathbb{R}^{m},
$$

where $\widetilde{D}$ is an arbitrary set such that $\bar{D} \cap\left[\left[t_{0}, t_{0}+T\right) \times \mathbb{R}^{n}\right] \subset \widetilde{D} \subset\left(-\infty, t_{0}+T\right) \times \mathbb{R}^{n}$, $w_{x}^{i}(t, x):=\operatorname{grad}_{x} w^{i}(t, x) \quad(i=1, \ldots, m)$ and $w_{x x}^{i}(t, x):=\left[\frac{\partial^{2} w^{i}(t, x)}{\partial x_{j} \partial x_{k}}\right]_{n \times n}(i=1, \ldots, m) . \mathrm{We}$ 
assume that the limits $w\left(t_{j}^{-}, x\right), w\left(t_{j^{+}}^{+}, x\right)(j=1, \ldots, s)$ exist for all admissible $x \in \mathbb{R}^{n}$, they are finite, all different and $w\left(t_{j}, x\right):=w\left(t_{j}^{+}, x\right)(j=1, \ldots, s)$ for all admissible $x \in \mathbb{R}^{n}$.

System (1.1) is studied together with impulsive and boundary inequalities. The impulsive inequalities are of the form

$$
u\left(t_{j}, x\right)-u\left(t_{j}^{-}, x\right) \leq v\left(t_{j}, x\right)-v\left(t_{j}^{-}, x\right) \quad(j=1, \ldots, s) .
$$

As a consequence of the theorem about the strong inequalities for system (1.1), we establish theorems about impulsive degenerate nonlinear parabolic differential inequalities and the uniqueness of a classical solution of an impulsive degenerate nonlinear parabolic differential problem.

The results obtained in the paper are direct generalizations of those given by the author in [2]. To prove the results of this paper, theorems of [2] are used. The paper is a continuation of author's publication [3] about impulsive parabolic problems. The impulsive conditions in the present paper are quite different from those considered in [3]. They are similar to the impulsive conditions used by Bainov, Kamont and Minchev in [1].

\section{Preliminaries}

We use the notation: $\mathbb{N}_{0}=\{0,1,2, \ldots\}, \mathbb{R}_{+}=[0, \infty)$. For any vectors $z=\left(z_{1}, \ldots, z_{m}\right) \in \mathbb{R}^{m}$, $\tilde{z}=\left(\widetilde{z}_{1}, \ldots, \widetilde{z}_{m}\right) \in \mathbb{R}^{m}$ we write $z \leq \widetilde{z}$ if $z_{i} \leq \widetilde{z}_{i}(i=1, \ldots, m)$.

By $\Omega$ we denote an arbitrary open subset of $\left(t_{0}, t_{0}+T\right) \times \mathbb{R}^{m}$, where $t_{0} \in \mathbb{R}$ and $T \in \mathbb{R}_{+} \backslash\{0\}$, such that the projection of $\Omega$ on the $t$-axis is the interval $\left(t_{0}, t_{0}+T\right)$.

Next, by $D$ we denote the subset of the set $\bar{\Omega} \cap\left[\left(t_{0}, t_{0}+T\right) \times \mathbb{R}^{n}\right]$ satisfying the condition that for any $(\widetilde{t}, \widetilde{x}) \in D$ there exists a number $\rho>0$ such that

$$
\left\{(t, x):(t-\tilde{t})^{2}+\sum_{i=1}^{n}\left(x_{i}-\tilde{x}_{i}\right)^{2}<\rho, \quad t<\tilde{t}\right\} \subset \Omega .
$$

It is clear that $\Omega \subset D$.

We define the sets

$$
S_{t}:=\left\{x \in \mathbb{R}^{n}:(t, x) \in \bar{D}\right\} \text { for } t \in\left[t_{0}, t_{0}+T\right]
$$

and

$$
\sigma_{t}:=\bar{D} \cap\left(\{t\} \times \mathbb{R}^{n}\right) \text { for } t \in\left[t_{0}, t_{0}+T\right]
$$

By $s$ we denote a fixed number belonging to $\mathbb{N}$.

Let $t_{1}, t_{2}, \ldots, t_{s}$ be arbitrary fixed real numbers such that

$$
t_{0}<t_{1}<\ldots<t_{s}<t_{0}+T
$$

We introduce the following sets:

$$
D_{j}:=D \cap\left[\left(t_{j}, t_{j+1}\right) \times \mathbb{R}^{n}\right] \quad(j=0,1, \ldots, s-1),
$$




$$
\begin{gathered}
D_{s}:=D \cap\left[\left(t_{s}, t_{0}+T\right) \times \mathbb{R}^{n}\right], \\
D_{*}:=\bigcup_{j=0}^{s} D_{j} \text { and } \sigma_{*}:=\bigcup_{j=1}^{s} \sigma_{t} .
\end{gathered}
$$

Let $\widetilde{D}$ be an arbitrary set such that

$$
\bar{D} \cap\left[\left[t_{0}, t_{0}+T\right) \times \mathbb{R}^{n}\right] \subset \widetilde{D} \subset\left(-\infty, t_{0}+T\right) \times \mathbb{R}^{n}, t_{0}+T \leq \infty .
$$

By $\Sigma$ we denote the part of $\partial D \backslash\left(\sigma_{t_{0}} \cup \sigma_{*} \cup \sigma_{t_{0}+T}\right)$ disjoint with $D$.

Assumption (A): For each $i \in\{1, \ldots, m\}$ let $\Sigma_{i}$ be a subset (possibly empty) of $\Sigma$ and let, for each $(t, x) \in \Sigma_{i}, \ell_{i}(t, x)$ be a direction. We assume that $\ell_{i}$ is orthogonal to the $t$-axis and some open segment, with one extremity at $(t, x)$, of the ray with origin at $(t, x)$ in the direction of $\ell_{i}$ is contained in $D$.

Given a subset $E$ of $\sigma_{t} \cup \sigma_{*} \cup \Sigma\left[E\right.$ of $\sigma_{*}$ ] and a function $\omega: D_{*} \rightarrow \mathbb{R}$, we say that $\omega$ has finite $t$-right-hand [t-left-hand] sided limits in $E \cup\{\infty\}$ if for every $(\widetilde{t}, \widetilde{x}) \in E$ and every $\widetilde{t} \in P(E)$, and for each sequence $\left(t^{\nu}, x^{\nu}\right) \in D_{*}$ such that $t^{\nu}>\tilde{t}\left[t^{\nu}<\tilde{t}\right], \quad t^{\nu} \rightarrow \tilde{t}$ and $\left(t^{\nu}, x^{\nu}\right) \rightarrow(\tilde{t}, \widetilde{x})$ or $\left|x^{\nu}\right| \rightarrow \infty$, the limit $\lim _{\nu \rightarrow \infty} \omega\left(t^{\nu}, x^{\nu}\right)$ is finite; here $P(E)$ is the projection of $E$ on the $t$-axis. Obviously, this limit does not depend on the choice of the sequence $\left(t^{\nu}, x^{\nu}\right)$ and it will be denoted by $\omega\left(\tilde{t}^{+}, \tilde{x}\right)$ and $\left.\omega \tilde{t}^{+}, \infty\right)\left[\omega\left(\tilde{t}^{-}, \tilde{x}\right)\right.$ and $\left.\omega\left(\tilde{t}^{-}, \infty\right)\right]$, respectively.

Let $E$ be a subset of $\sigma_{t_{0}} \cup \sigma_{*} \cup \Sigma$. If for $\tilde{t} \in P(E)$ there is a sequence $\left(t^{\nu}, x^{\nu}\right) \in D_{*}$ such that $t^{\nu}>\tilde{t}, t^{\nu} \rightarrow \tilde{t}$ and $\left|x^{\nu}\right| \rightarrow \infty$ then we denote by $(\tilde{t}, \infty)$ the class of all such sequences. By a function $\varphi: E \cup\{\infty\} \rightarrow \mathbb{R}$ we mean a function defined for $(t, x) \in E$ and $(t, \infty)$ with $t \in P(E)$.

By $P C_{m}(\widetilde{D})$ we denote the space of mappings

$$
w: \widetilde{D} \ni(t, x) \rightarrow w(t, x)=\left(w^{1}(t, x), \ldots, w^{m}(t, x)\right) \in \mathbb{R}^{m},
$$

such that, for every $i \in\{1, \ldots, m\}, w^{i}$ is continuous in $\left(D \cup \Sigma_{i}\right) \backslash \sigma_{*}$, has finite $t$-right-hand sided limits $w^{i}\left(t^{+}, x\right), w^{i}\left(t^{+}, \infty\right)$ in $\sigma_{t_{0}} \cup \sigma_{*} \cup\left(\Sigma \backslash \Sigma_{i}\right) \cup\{\infty\}$, has finite $t$-left-hand sided limits $w^{i}\left(t^{-}, x\right), w^{i}\left(t^{-}, \infty\right)$ in $\sigma_{*} \cup\{\infty\}$, and $w^{i}(t, x):=w^{i}\left(t^{+}, x\right)$ for $(t, x) \in \sigma_{t_{0}} \cup \sigma_{*} \cup\left(\Sigma \backslash \Sigma_{i}\right)$ and $w^{i}(t, \infty):=w^{i}\left(t^{+}, \infty\right)$ for $t \in P\left[\sigma_{t_{0}} \cup \sigma_{*} \cup\left(\Sigma \backslash \Sigma_{i}\right)\right]$.

For $w, \widetilde{w} \in P C_{m}(\widetilde{D})$ and for every fixed $t<t_{0}+T$, we write $w \stackrel{t}{\leq} \widetilde{w}$ if $w^{i}(\tau, x) \leq \widetilde{w}^{i}(\tau, x)$ for $(\tau, x) \in \widetilde{D}, \tau \leq t \quad(i=1, \ldots, m)$. Given the sets $\Sigma_{i}(i=1, \ldots, m)$ and the directions $\ell_{i}(i=1, \ldots$, $m$ ) satisfying Assumption (A), a function $w \in P C_{m}(\widetilde{D})$ is said to belong to $P C_{m, \Sigma}^{1,2}(\widetilde{D})$ if $w_{t}^{i}, w_{x}^{i}, w_{x x}^{i}(i=1, \ldots, m)$ are continuous in $D_{*}$ and the derivatives $\frac{d w^{i}}{d \ell_{i}} \quad(i=1, \ldots, m)$ are finite on $\Sigma_{i}(i=1, \ldots, m)$, respectively.

By $M_{n \times n}(\mathbb{R})$ we denote the space of real square symmetric matrices $r=\left[r_{j k}\right]_{n \times n}$. For each $i \in\{1, \ldots, m\}$ by $F_{i}$ we denote the mapping

$$
\begin{gathered}
F_{i}: D_{*} \times \mathbb{R}^{m} \times \mathbb{R} \times \mathbb{R}^{n} \times M_{n \times n}(\mathbb{R}) \times P C_{m, \Sigma}^{1,2}(\widetilde{D}) \\
\ni(t, x, z, p, q, r, w) \rightarrow F_{i}(t, x, z, p, q, r, w) \in \mathbb{R}
\end{gathered}
$$

where $q=\left(q_{1}, \ldots, q_{n}\right)$ and $r=\left[r_{j k}\right]_{n \times n}$.

We use the notation

$$
F_{i}[t, x, w]:=F_{i}\left(t, x, w(t, x), w_{t}^{i}(t, x), w_{x}^{i}(t, x), w_{x x}^{i}(t, x), w\right) \quad(i=1, \ldots, m)
$$


for all $(t, x) \in D_{*}$ and $w \in P C_{m, \Sigma}^{1,2}(\widetilde{D})$.

By $Z$ we denote a fixed subset of $P C_{m, \Sigma}^{1,2}(\widetilde{D})$. Functions $u$ and $v$ belonging to $Z$ are called solutions of the system

$$
F_{i}[t, x, u]>F_{i}[t, x, v] \quad(i=1, \ldots, m)
$$

in $D_{*}$, if they satisfy $(2.1)$ for all $(t, x) \in D_{*}$.

The functions $F_{i}(i=1, \ldots, m)$ are said to be parabolic with respect to $w \in P C_{m, \Sigma}^{1,2}(\widetilde{D})$ in $D_{*}$ if for every $r=\left[r_{j k}\right], \tilde{r}=\left[\tilde{r}_{j k}\right] \in M_{n \times n}(\mathbb{R})$ and $(t, x) \in D_{*}$ the following implications hold:

$$
\begin{gathered}
r \leq \tilde{r} \Rightarrow F_{i}\left(t, x, u(t, x), u_{t}^{i}(t, x), u_{x}^{i}(t, x), r, u\right) \\
\leq F_{i}\left(t, x, u(t, x), u_{t}^{i}(t, x), u_{x}^{i}(t, x), \tilde{r}, u\right) \quad(i=1, \ldots, m),
\end{gathered}
$$

where $r \leq \tilde{r}$ means that the inequality $\sum_{j, k=1}^{n}\left(r_{j k}-\widetilde{r}_{j k}\right) \lambda_{j} \lambda_{k} \leq 0$ is satisfied for each
$\left(\lambda_{1}, \ldots, \lambda_{n}\right) \in \mathbb{R}^{n}$.

\section{Theorem about Impulsive Functional-Differential Inequalities}

Theorem 3.1. Assume that:

1. The functions $F_{i}(i=1, \ldots, m)$ are weakly increasing with respect to $z_{1}, \ldots, z_{i-1}$, $z_{i+1}, \ldots, z_{m}(i=1, \ldots, m)$, respectively. Moreover, $F_{i}(i=1, \ldots, m)$ are weakly increasing with respect to $w$ in the sense of the relation $\stackrel{t}{\leq}$ for all $t \in\left(t_{0}, t_{0}+T\right)$ and

$$
F_{i}(t, x, z, p, q, r, w) \geq F_{i}(t, x, z, \tilde{p}, q, r, w) \quad(i=1, \ldots, m)
$$

for all $(t, x) \in D_{*}, z \in \mathbb{R}^{m}, p<\tilde{p}, q \in \mathbb{R}^{n}, r \in M_{n \times n}(\mathbb{R}), w \in Z$.

2. For the given sets $\Sigma_{i}(i=1, \ldots, m)$ and the directions $\ell_{i} \quad(i=1, \ldots, m)$ satisfying Assumption $(A)$, for the given functions $a_{i}: \Sigma_{i} \rightarrow \mathbb{R}_{+}(i=1, \ldots, m)$ and for the given functions $\phi_{i}: \Sigma_{i} \times \mathbb{R} \rightarrow \mathbb{R}(i=1, \ldots, m)$ of the variables $(t, x, \xi)$ and weakly increasing with respect to $\xi$, functions $u$ and $v$ belonging to $Z$ satisfy the inequalities

$$
\begin{gathered}
u(t, x)<v(t, x) \text { for }(t, x) \in \widetilde{D} \backslash \bar{D}, \\
u^{i}(t, x)<v^{i}(t, x) \text { for }(t, x) \in \sigma_{t_{0}} \cup\left(\Sigma \backslash \Sigma_{i}\right) \cup\{\infty\} \quad(i=1, \ldots, m), \\
u(t, x)-u\left(t^{-}, x\right)<v(t, x)-v\left(t^{-}, x\right) \text { for }(t, x) \in \sigma_{*}, \\
\phi_{i}\left(t, x, u^{i}(t, x)\right)-\phi_{i}\left(t, x, v^{i}(t, x)\right)<a_{i}(t, x) \frac{d\left[u^{i}(t, x)-v^{i}(t, x)\right]}{d \ell_{i}} \\
\text { for }(t, x) \in \Sigma_{i} \quad(i=1, \ldots, m)
\end{gathered}
$$

and the condition

$$
u^{i}(t, x) \neq v^{i}(t, x) \text { for }(t, x) \in \Sigma_{i} \quad(i=1, \ldots, m) .
$$


3. $\quad F_{i} \quad(i=1, \ldots, m)$ are parabolic with respect to $u$ in $D_{*}$ and $u, v$ are solutions of system $(2.1)$ in $D_{*}$.

Then,

$$
u(t, x)<v(t, x) \text { for }(t, x) \in \tilde{D}
$$

Proof. To prove Theorem 3.1 consider the following problem:

$$
\begin{gathered}
F_{i}[t, x, u]>F_{i}[t, x, v] \text { for }(t, x) \in D_{0}(i=1, \ldots, m), \\
u(t, x)<v(t, x) \text { for }(t, x) \in(\widetilde{D} \backslash \bar{D}) \cap\left(\left(-\infty, t_{1}\right) \times \mathbb{R}^{n}\right), \\
u^{i}(t, x)<v^{i}(t, x) \text { for }(t, x) \in\left[\sigma_{t_{0}} \cup\left(\Sigma \backslash \Sigma_{i}\right) \cup\{\infty\}\right] \\
\cap\left[\left[t_{0}, t_{1}\right) \times \mathbb{R}^{n}\right](i=1, \ldots, m), \\
\phi_{i}\left(t, x, u^{i}(t, x)\right)-\phi_{i}\left(t, x, v^{i}(t, x)\right)<a_{i}(t, x) \frac{d\left[u^{i}(t, x)-v^{i}(t, x)\right]}{d \ell_{i}} \\
\text { for }(t, x) \in \Sigma_{i} \cap\left[\left(t_{0}, t_{1}\right) \times \mathbb{R}^{n}\right] \quad(i=1, \ldots, m) .
\end{gathered}
$$

According to the assumptions of Theorem 3.1 corresponding to problem (3.7), by Theorem 2.1 from [2] applied to set $D_{0}$, we obtain the inequality

$$
u(t, x)<v(t, x) \text { for }(t, x) \in D_{0} .
$$

By (3.8) and by the fact that $u, v \in P C_{m}(\widetilde{D})$,

$$
u\left(t^{-}, x\right) \leq v\left(t^{-}, x\right) \text { for }(t, x) \in \sigma_{t_{1}} .
$$

From (3.3) and (3.9), we have

$$
u(t, x)<v(t, x) \text { for }(t, x) \in \sigma_{t_{1}} .
$$

Inequalities (3.1), (3.8), (3.2) and (3.10) imply that

$$
u^{i}(t, x)<v^{i}(t, x) \text { for }(t, x) \in\left(\widetilde{D} \backslash \Sigma_{i}\right) \cap\left[\left(-\infty, t_{1}\right] \times \mathbb{R}^{n}\right] \quad(i=1, \ldots, m) .
$$

By $(3.11),(3.5)$ and the fact that $u^{i}(i=1, \ldots, m)$ is continuous in $\Sigma_{i}(i=1, \ldots, m)$, we get

$$
u(t, x)<v(t, x) \text { for }(t, x) \in \widetilde{D} \cap\left[\left(-\infty, t_{1}\right] \times \mathbb{R}^{n}\right]
$$

Now, set the following problem: 


$$
\begin{gathered}
F_{i}[t, x, u]>F_{i}[t, x, v] \text { for }(t, x) \in D_{1} \quad(i=1, \ldots, m), \\
u(t, x)<v(t, x) \text { for }(t, x) \in\left[\widetilde{D} \cap\left(\left(-\infty, t_{2}\right) \times \mathbb{R}^{n}\right)\right] \backslash \bar{D}_{1}, \\
u^{i}(t, x)<v^{i}(t, x) \text { for }(t, x) \in\left[\sigma_{t_{1}} \cup\left(\Sigma \backslash \Sigma_{i}\right) \cup\{\infty\}\right] \\
\cap\left[\left[t_{1}, t_{2}\right) \times \mathbb{R}^{n}\right](i=1, \ldots, m), \\
\phi_{i}\left(t, x, u^{i}(t, x)\right)-\phi_{i}\left(t, x, v^{i}(t, x)\right)<a_{i}(t, x) \frac{d\left[u^{i}(t, x)-v^{i}(t, x)\right]}{d \ell_{i}} \\
\text { for }(t, x) \in \Sigma_{i} \cap\left[\left(t_{1}, t_{2}\right) \times \mathbb{R}^{n}\right] \quad(i=1, \ldots, m) .
\end{gathered}
$$

According to the assumptions of Theorem 3.1 corresponding to problem (3.13), by Theorem 2.1 from [2] applied to set $D_{1}$, we arrive at the inequality

$$
u(t, x)<v(t, x) \text { for }(t, x) \in D_{1}
$$

By (3.14) and by the fact that $u, v \in P C_{m}(\tilde{D})$,

$$
u\left(t^{-}, x\right) \leq v\left(t^{-}, x\right) \text { for }(t, x) \in \sigma_{t_{2}}
$$

From (3.3) and (3.15), we have

$$
u(t, x)<v(t, x) \text { for }(t, x) \in \sigma_{t_{2}} .
$$

Inequalities (3.1), (3.12), (3.14), (3.2) and (3.16) imply that

$$
u^{i}(t, x)<v^{i}(t, x) \text { for }(t, x) \in\left[\widetilde{D} \cap\left[\left(-\infty, t_{2}\right] \times \mathbb{R}^{n}\right]\right] \backslash\left[\Sigma_{i} \cap\left[\left(t_{1}, t_{2}\right) \times \mathbb{R}^{n}\right]\right] \text {. }
$$

By $(3.17),(3.5)$ and the fact that $u^{i}(i=1, \ldots, m)$ is continuous in $\Sigma_{i}(i=1, \ldots, m)$, we get

$$
u(t, x)<v(t, x) \text { for }(t, x) \in \widetilde{D} \cap\left[\left(-\infty, t_{2}\right] \times \mathbb{R}^{n}\right]
$$

Repeating the above procedure $s-2$ times, we obtain

$$
u(t, x)<v(t, x) \text { for }(t, x) \in \sigma_{t_{s}}
$$

and

$$
u(t, x)<v(t, x) \text { for }(t, x) \in \widetilde{D} \cap\left[\left(-\infty, t_{s}\right] \times \mathbb{R}^{n}\right]
$$

Finally, consider the problem 


$$
\begin{gathered}
F_{i}[t, x, u]>F_{i}[t, x, v] \text { for }(t, x) \in D_{s}(i=1, \ldots, m), \\
u(t, x)<v(t, x) \text { for }(t, x) \in \widetilde{D} \backslash \bar{D}_{s}, \\
u^{i}(t, x)<v^{i}(t, x) \text { for }(t, x) \in\left[\sigma_{t_{s}} \cup\left(\Sigma \backslash \Sigma_{i}\right) \cup\{\infty\}\right] \\
\cap\left[\left[t_{s}, t_{0}+T\right) \times \mathbb{R}^{n}\right] \quad(i=1, \ldots, m), \\
\phi_{i}\left(t, x, u^{i}(t, x)\right)-\phi_{i}\left(t, x, v^{i}(t, x)\right)<a_{i}(t, x) \frac{d\left[u^{i}(t, x)-v^{i}(t, x)\right]}{d \ell_{i}} \\
\text { for }(t, x) \in \Sigma_{i} \cap\left[\left(t_{s}, t_{0}+T\right) \times \mathbb{R}^{n}\right] \quad(i=1, \ldots, m) .
\end{gathered}
$$

According to the assumptions of Theorem 3.1 corresponding to problem (3.21), by Theorem 2.1 from [2] applied to set $D_{s}$, we get the inequality

$$
u(t, x)<v(t, x) \text { for }(t, x) \in D_{s} .
$$

Inequalities (3.1), (3.20), (3.22), (3.2) and (3.19) imply that

$$
u^{i}(t, x)<v^{i}(t, x) \text { for }(t, x) \in \widetilde{D} \backslash\left[\Sigma_{i} \cap\left[\left(t_{s}, t_{0}+T\right) \times \mathbb{R}^{n}\right]\right](i=1, \ldots, m) .
$$

By $(3.23),(3.5)$ and the fact that $u^{i}(i=1, \ldots, m)$ is continuous in $\Sigma_{i}(i=1, \ldots, m)$, we have

$$
u(t, x)<v(t, x) \text { for }(t, x) \in \tilde{D} .
$$

\section{Theorems about Impulsive Differential Inequalities}

From the proof of Theorem 3.1 it is easy to see that the following theorem is true:

Theorem 4.1. Assume that:

1. $\widetilde{D}=\bar{D}$ and the functions

$$
G_{i}: D_{*} \times \mathbb{R}^{m} \times \mathbb{R} \times \mathbb{R}^{n} \times M_{n \times n}(\mathbb{R}) \ni(t, x, z, p, q, r) \rightarrow G_{i}(t, x, z, p, q, r) \in \mathbb{R}
$$

are weakly increasing with respect to $z_{1}, \ldots, z_{i-1}, z_{i+1}, \ldots, z_{m}(i=1, \ldots, m)$, respectively, and

$$
G_{i}(t, x, z, p, q, r) \geq G_{i}(t, x, z, \tilde{p}, q, r) \quad(i=1, \ldots, m)
$$

for all $(t, x) \in D_{*}, z \in \mathbb{R}^{m}, p<\tilde{p}, q \in \mathbb{R}^{n}, r \in M_{n \times n}(\mathbb{R})$.

2. For the given sets $\Sigma_{i}(i=1, \ldots, m)$ and the directions $\ell_{i}(i=1, \ldots, m)$ satisfying Assumption $(A)$, for the given functions $a_{i}: \Sigma_{i} \rightarrow \mathbb{R}_{+}(i=1, \ldots, m)$ and for the given functions $\phi_{i}: \Sigma_{i} \times \mathbb{R} \rightarrow \mathbb{R} \quad(i=1, \ldots, m)$ of the variables $(t, x, \xi)$ and weakly increasing with respect to $\xi$, functions $u$ and $v$ belonging to $Z \subset P C_{m, \Sigma}^{1,2}(\bar{D})$ satisfy inequalities $(3.2)-(3.4)$.

3. $G_{i}(i=1, \ldots, m)$ are parabolic with respect to $u$ in $D_{*}$, and $u, v$ are solutions of the system

$$
G_{i}\left(t, x, u(t, x), u_{t}^{i}(t, x), u_{x}^{i}(t, x), u_{x x}^{i}(t, x)\right)
$$




$$
>G_{i}\left(t, x, v(t, x), v_{t}^{i}(t, x), v_{x}^{i}(t, x), v_{x x}^{i}(t, x)\right) \quad(i=1, \ldots, m)
$$

in $D_{*}$.

Then

$$
u^{i}(t, x)<v^{i}(t, x) \text { for }(t, x) \in\left(\bar{D} \backslash \Sigma_{i}\right) \cap\left(\left[t_{0}, t_{0}+T\right) \times \mathbb{R}^{n}\right) \quad(i=1, \ldots, m)
$$

and

$$
u^{i}(t, x) \leq v^{i}(t, x) \text { for }(t, x) \in \Sigma_{i} \quad(i=1, \ldots, m) .
$$

Moreover,

(iii)

$$
u(t, x)<v(t, x) \text { for }(t, x) \in \bar{D} \cap\left(\left[t_{0}, t_{0}+T\right) \times \mathbb{R}^{n}\right)
$$

if (3.5) holds.

As a consequence of Theorem 4.1, we obtain the following theorem:

Theorem 4.2. Assume that:

1. $\widetilde{D}=\bar{D}$ and the function

$$
G: D_{*} \times \mathbb{R} \times \mathbb{R} \times \mathbb{R}^{n} \times M_{n \times n}(\mathbb{R}) \ni(t, x, z, p, q, r) \rightarrow G(t, x, z, p, q, r) \in \mathbb{R}
$$

is weakly decreasing with respect to $z$ and $p$ in $D_{*}$.

2. For the given set $\widehat{\Sigma} \subset \Sigma$ and the direction $\ell$ satisfying Assumption $(A)$, for the given function a: $\widehat{\Sigma} \rightarrow \mathbb{R}+$ and for the given function $\phi: \widehat{\Sigma} \times \mathbb{R} \rightarrow \mathbb{R}$ of the variables $(t, x, \xi)$ and strictly increasing with respect to $\xi$, functions $u$ and $v$ belonging to $Z \subset P C_{1, \Sigma}^{1,2}(\bar{D})$ satisfy the inequalities

$$
\begin{gathered}
u(t, x) \leq v(t, x) \text { for }(t, x) \in \sigma_{t_{0}} \cup(\Sigma \backslash \widehat{\Sigma}) \cup\{\infty\}, \\
u(t, x)-u\left(t^{-}, x\right) \leq v(t, x)-v\left(t^{-}, x\right) \text { for }(t, x) \in \sigma_{*}
\end{gathered}
$$

and

$$
\phi(t, x, u(t, x))-\phi(t, x, v(t, x)) \leq a(t, x) \frac{d[u(t, x)-v(t, x)]}{d \ell} \text { for }(t, x) \in \widehat{\Sigma} .
$$

3. $\quad G$ is parabolic with respect to $u$ in $D_{*}$ and $u, v$ are solutions of the inequality

in $D_{*}$.

$$
\begin{aligned}
& G\left(t, x, u(t, x), u_{t}(t, x), u_{x}(t, x), u_{x x}(t, x)\right) \\
& >G\left(t, x, v(t, x), v_{t}(t, x), v_{x}(t, x), v_{x x}(t, x)\right)
\end{aligned}
$$

Then

$$
u(t, x) \leq v(t, x) \text { for }(t, x) \in D_{*} .
$$

Proof. Let $\epsilon>0$ and let

$$
v^{\epsilon}(t, x):=\left\{\begin{array}{c}
v(t, x)+\epsilon \text { for }(t, x) \in \bar{D}_{0} \backslash \sigma_{t_{1}}, \\
v(t, x)+2 \epsilon \text { for }(t, x) \in \bar{D}_{1} \backslash \sigma_{t_{2}} \\
\ldots \ldots \ldots \ldots \ldots \ldots \\
v(t, x)+s \epsilon \text { for }(t, x) \in \bar{D}_{s-1} \backslash \sigma_{t_{s}} \\
v(t, x)+(s+1) \epsilon \text { for }(t, x) \in \bar{D}_{s} \backslash \sigma_{t_{0}}+T
\end{array}\right.
$$


By (4.1), (4.3) and by the fact that $G$ is weakly decreasing with respect to $z$, we obtain

$$
\begin{gathered}
G\left(t, x, u(t, x), u_{t}(t, x), u_{x}(t, x), u_{x x}(t, x)\right) \\
-G\left(t, x, v^{\epsilon}(t, x), v_{t}^{\epsilon}(t, x), v_{x}^{\epsilon}(t, x), v_{x x}^{\epsilon}(t, x)\right) \\
>G\left(t, x, v(t, x), v_{t}(t, x), v_{x}(t, x), v_{x x}(t, x)\right) \\
-G\left(t, x, v^{\epsilon}(t, x), v_{t}^{\epsilon}(t, x), v_{x}^{\epsilon}(t, x), v_{x x}^{\epsilon}(t, x)\right) \\
\geq 0 \text { for }(t, x) \in D_{*} .
\end{gathered}
$$

Moreover, from assumption 2 of Theorem 4.2 and from (4.3) it follows that

$$
\begin{gathered}
u(t, x)<v^{\epsilon}(t, x) \text { for }(t, x) \in \sigma_{t_{0}} \cup(\Sigma \backslash \widehat{\Sigma}) \cup\{\infty\}, \\
\phi(t, x, u(t, x))-\phi\left(t, x, v^{\epsilon}(t, x)\right) \\
<\phi(t, x, u(t, x))-\phi(t, x, v(t, x)) \\
\leq a(t, x) \frac{d[u(t, x)-v(t, x)]}{d \ell} \\
=a(t, x) \frac{d\left[u(t, x)-v^{\epsilon}(t, x)\right]}{d \ell} \text { for }(t, x) \in \widehat{\Sigma}
\end{gathered}
$$

and

$$
\begin{gathered}
u\left(t_{j}, x\right)-u\left(t_{j}^{-}, x\right) \leq v\left(t_{j}, x\right)-v\left(t_{j}^{-}, x\right) \\
<\left[v\left(t_{j}, x\right)+(j+1) \epsilon\right]-\left[v\left(t_{j}^{-}, x\right)+j \epsilon\right] \\
=v^{\epsilon}\left(t_{j}, x\right)-v^{\epsilon}\left(t_{j}^{-}, x\right) \text { for } x \in S_{t_{j}}(j=1,2, \ldots, s) .
\end{gathered}
$$

Then we have the inequality

$$
u(t, x)<v^{\epsilon}(t, x) \text { for }(t, x) \in D_{*}
$$

because functions $u$ and $v^{\epsilon}$ satisfy all the assumptions of Theorem 4.1. Hence (4.2) holds.

Remark 4.1. From the proof of Theorem 4.2 it is easy to see that if function $G$ from Theorem 4.2 is strictly decreasing with respect to $z$ and weakly decreasing with respect to $p$ in $D_{*}$ then Theorem 4.2 is true if strong inequality (4.1) is replaced by the weak inequality

$$
\begin{gathered}
G\left(t, x, u(t, x), u_{t}(t, x), u_{x}(t, x), u_{x x}(t, x)\right) \\
\geq G\left(t, x, v(t, x), v_{t}(t, x), v_{x}(t, x), v_{x x}(t, x)\right), \quad(t, x) \in D_{*} .
\end{gathered}
$$

Theorem 4.2 and Remark 4.1 imply the following theorem about the uniqueness of a classical solution of a mixed impulsive parabolic differential problem: 
Theorem 4.3. Assume that:

1. $\widetilde{D}=\bar{D}$ and the function $G$ from Theorem 4.2 is strictly decreasing with respect to $z$ and weakly decreasing with respect to $p$ in $D_{*}$.

2. The set $\widehat{\Sigma} \subset \Sigma$ and the direction $\ell$ satisfy Assumption $(A), a: \widehat{\Sigma} \rightarrow \mathbb{R}_{+}$is a given function, the function $\phi: \widehat{\Sigma} \times \mathbb{R} \rightarrow \mathbb{R}$ of the variables $(t, x, \xi)$ is strictly increasing with respect to $\xi$, and $f: \sigma_{t_{0}} \cup(\Sigma \backslash \widehat{\Sigma}) \cup\{\infty\} \rightarrow \mathbb{R}, g: \sigma_{*} \rightarrow \mathbb{R}, h: \widehat{\Sigma} \rightarrow \mathbb{R}$ are given functions.

Then in the class of all functions $w$ belonging to $P C_{1, \Sigma}^{1,2}(\bar{D})$ and such that function $G$ is parabolic with respect to $w$ in $D_{*}$ there exists at most one function satisfying the following mixed impulsive parabolic differential problem:

$$
\begin{gathered}
G\left(t, x, w(t, x), w_{t}(t, x), w_{x}(t, x), w_{x x}(t, x)\right)=0, \quad(t, x) \in D_{*}, \\
w(t, x)=f(t, x), \quad(t, x) \in \sigma_{t_{0}} \cup(\Sigma \backslash \widehat{\Sigma}) \cup\{\infty\}, \\
w(t, x)-w\left(t^{-}, x\right)=g(t, x),(t, x) \in \sigma_{*}, \\
g(t, x, w(t, x))-a(t, x) \frac{d w(t, x)}{d \ell}=h(t, x), \quad(t, x) \in \widehat{\Sigma} .
\end{gathered}
$$

\section{Remarks}

Remark 5.1. Since the functions $F_{i}(i=1, \ldots, m)$ from Theorem 3.1 are weakly decreasing with respect to $p$ then these functions may be, particularly, defined by the following formulae:

$$
F_{i}(t, x, z, p, q, r, w):=f_{i}(t, x, z, q, r, w)-c_{i}(t, x) p \quad(i=1, \ldots, m)
$$

where $(t, x) \in D_{*}, z \in \mathbb{R}^{m}, p \in \mathbb{R}, q \in \mathbb{R}^{n}, \quad r \in M_{n \times n}(\mathbb{R}), w \in Z$, and $c_{i}(t, x) \geq 0(i=1, \ldots, m)$ for $(t, x) \in D_{*}$.

The same remarks are true for functions $G_{i}(i=1, \ldots, m)$ and $G$ from Theorems 4.1-4.3.

Therefore, the degenerate parabolic problems from this paper are more general than the parabolic problems, in the normal form with respect to $p$, corresponding to the considered degenerate parabolic problems.

Remark 5.2. Theorems 4.2 and 4.3 are formulated only for the differential parabolic problems and for $m=1$ because assuming, simultaneously, that $F_{i}(i=1, \ldots, m)$ from Theorem 3.1 are weakly increasing with respect to $z_{1}, \ldots, z_{i-1}, z_{i+1}, \ldots, z_{n}, w$ and weakly decreasing with respect to $z_{1}, \ldots, z_{n}, w$ we can consider only the differential problems, where $m=1$.

\section{References}

[1] Bainov, D., Kamont, Z. and Minchev, E., On first order impulsive partial differential inequalities, Appl. Math. Comp. (1994), (to appear).

[2] Byszewski, L., On degenerate nonlinear parabolic functional-differential inequalities in arbitrary domains, Univ. Iagell. Acta Math. 24 (1984), 341-348.

[3] Byszewski, L., Impulsive degenerate nonlinear parabolic functional-differential inequalities, J. Math. Anal. Appl. 164.2 (1992), 549-559. 


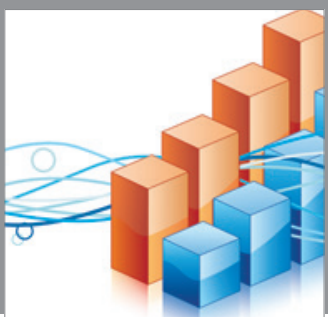

Advances in

Operations Research

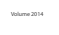

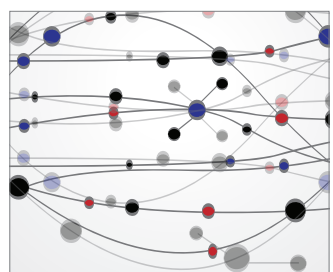

\section{The Scientific} World Journal
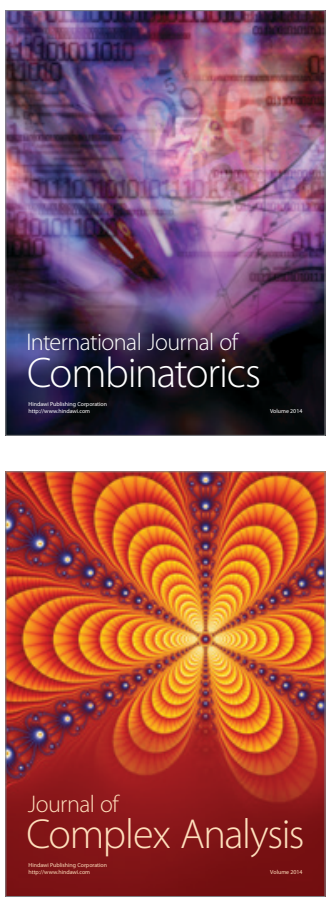

International Journal of

Mathematics and

Mathematical

Sciences
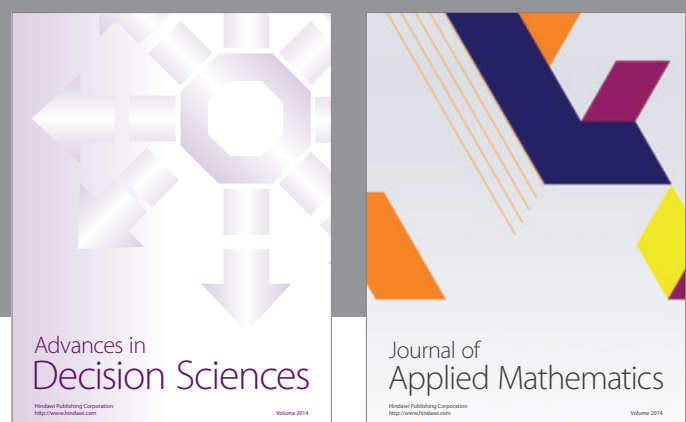

Journal of

Applied Mathematics
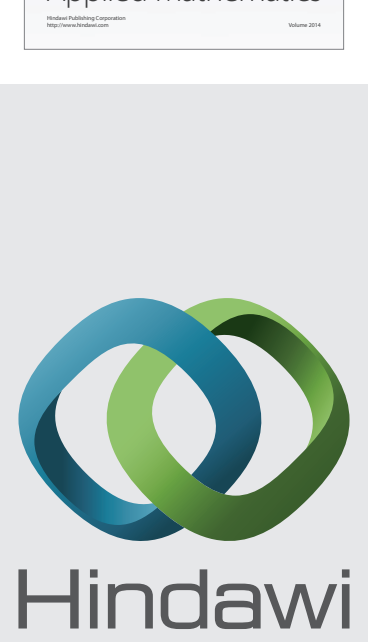

Submit your manuscripts at http://www.hindawi.com
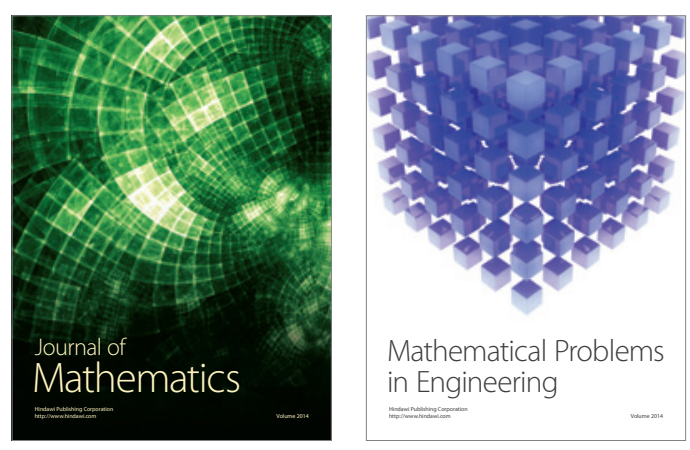

Mathematical Problems in Engineering
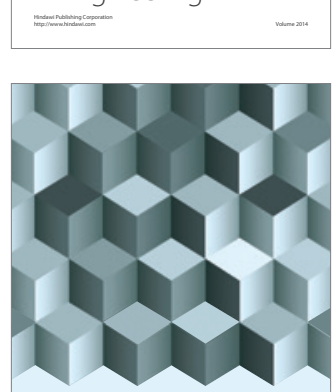

Journal of

Function Spaces
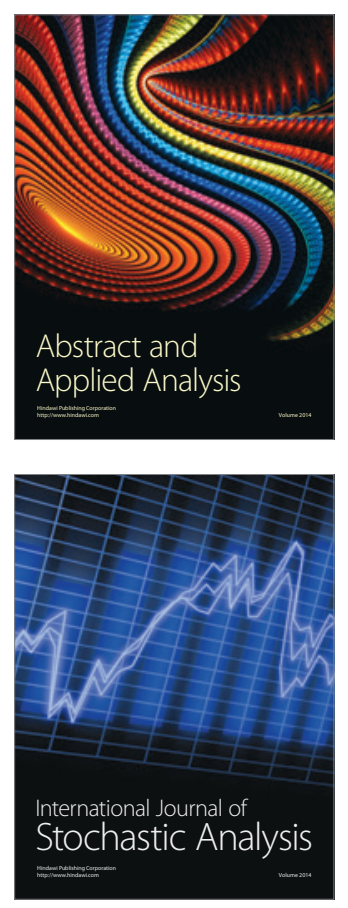

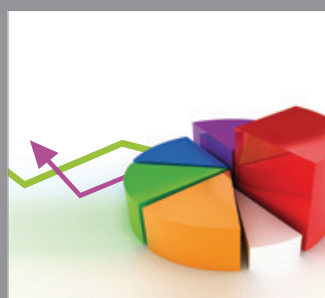

ournal of

Probability and Statistics

Promensencen
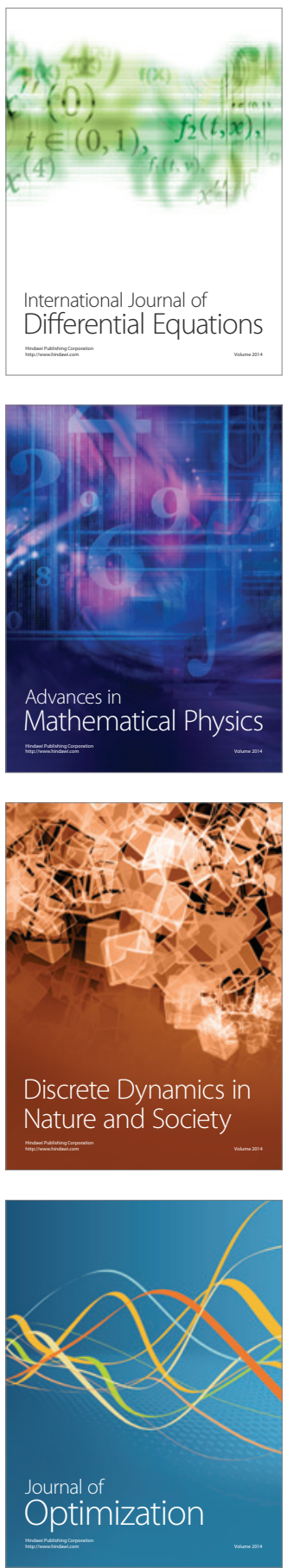